\title{
Software Defined Network (SDN) and OpenFlow Protocol in 5G Network
}

\author{
Khaled Alghamdi' ${ }^{1}$ Robin Braun ${ }^{2}$ \\ ${ }^{1}$ School of Electrical and Data Engineering, University of Technology, Sydney, Australia \\ ${ }^{2}$ College of Computer Science and Information Technology, Al Baha University, Al Baha, Saudi Arabia \\ Email: kyadg903@hotmail.com, robin.braun@uts.edu.au, alghamdi.kh@bu.edu.sa
}

How to cite this paper: Alghamdi, K. and Braun, R. (2020) Software Defined Network (SDN) and OpenFlow Protocol in 5G Network. Communications and Network, 12, $28-40$.

https://doi.org/10.4236/cn.2020.121002

Received: December 9, 2019

Accepted: January 13, 2020

Published: January 16, 2020

Copyright $\odot 2020$ by author(s) and Scientific Research Publishing Inc. This work is licensed under the Creative Commons Attribution International License (CC BY 4.0).

http://creativecommons.org/licenses/by/4.0/

\begin{abstract}
The world is moving at a high speed in the implementation and innovations of new systems and gadgets. $3 \mathrm{G}$ and $4 \mathrm{G}$ networks support currently wireless network communications. However, the networks are deemed to be slow and fail to receive signals or data transmission to various regions as a result of solving the problem. This paper will analyze the use of Software Defined Network (SDN) in a 5G (fifth generation) network that can be faster and reliable. Further, in Mobile IP, there exist triangulation problems between the sending and receiving nodes along with latency issues during handoff for the mobile nodes causing huge burden in the network. With Cloud Computing and ecosystem for Virtualization developed for the Core and Radio Networks SDN OpenFlow seems to be a seamless solution for determining signal flow between mobiles. There have been a lot of researches going on for deploying SDN OpenFlow with the 5G Cellular Network. The current paper performs benchmarks as a feasibility need for implementing SDN OpenFlow for 5G Cellular Network. The Handoff mechanism impacts the scalability required for a cellular network and simulation results can be further used to be deployed the 5 G Network.
\end{abstract}

\section{Keywords}

SDN, LTE, Mobile IP, LTE Advanced, OpenFlow, 5G

\section{Introduction}

Mobile IP (or MIP) as defined in [1] is a protocol based on the standards specified in RFC 5944 for mobile devices where its IP addresses are maintained as permanent as mobile subscribers move within different networks.

IETF RFC 5944 and IETF RFC 4721 describe the Mobile IPv4 implementa- 
tions. Further, for the next generation mobility implementation Internet Protocol IPv6 is based on the standards specified in the RFC 6275.

In a cellular environment like LTE where there are billions of Mobile Nodes and real-time traffic, the Mobile IP suffers from several issues and gaps which include lack of support for paging, large signaling load at global level and high update latency during handoff and triangular routing.

This can be also seen with other Mobile IP technologies such as Mobile IPv4 and Mobile IPv6.

An SDN Controller is a part of the control plane of the SDN Architecture. This can be further understood with elaboration from [2] [3] with Figure 1 as follows.

Here, the A-CPI and D-CPI are the reference points for interfaces of the SDN Controller with the SDN Application and Data plane respectively. An SDN controller can also develop its capability to communicate with the non-SDN plane and other peer SDN Controllers. A subordinate SDN Controller can also be further developed. The agent here virtualizes and shares the resources underlying it. The agent also at different level of abstraction exposes control over the network. There can be multiple agents for a Network Element in a SDN controller. This leads to developing a use case for the SDN provider as with the reclusive virtualized network.

The requirement for recursion implies a hierarchical virtual service of the SDN controllers and includes intermediate interfaces called the I-CPI.

In this paper, we will review a simulation scenario for Hand Off Mechanism with SDN controller giving its advantage of deployment in the 5G Network validating the SDN NFV Ecosystem for the Radio Network Management and Core Network Architecture.

However, there are limitations with the Mobile IP which has its own challenges for implementing in the network which includes Security issues like Denial-of-Service attack, Theft of information Passive Eaves Dropping, insider attack, etc.

\section{Software Defined Network (SDN)}

A software-defined network is a communication device under the networking section that controls the functionality of the networking devices and makes a separation of data.

The networking devices that are controlled by a software Defined network include packet switches, routers, and local area network (LAN) switches. The devices must have well-defined application programming interface (API) between two connected devises.

The devices used under networking in big organizations are routers, networks, and other networking devices that encompass the control functions and data making it difficult to alter the network infrastructure within a system [4] (see Figure 2). 


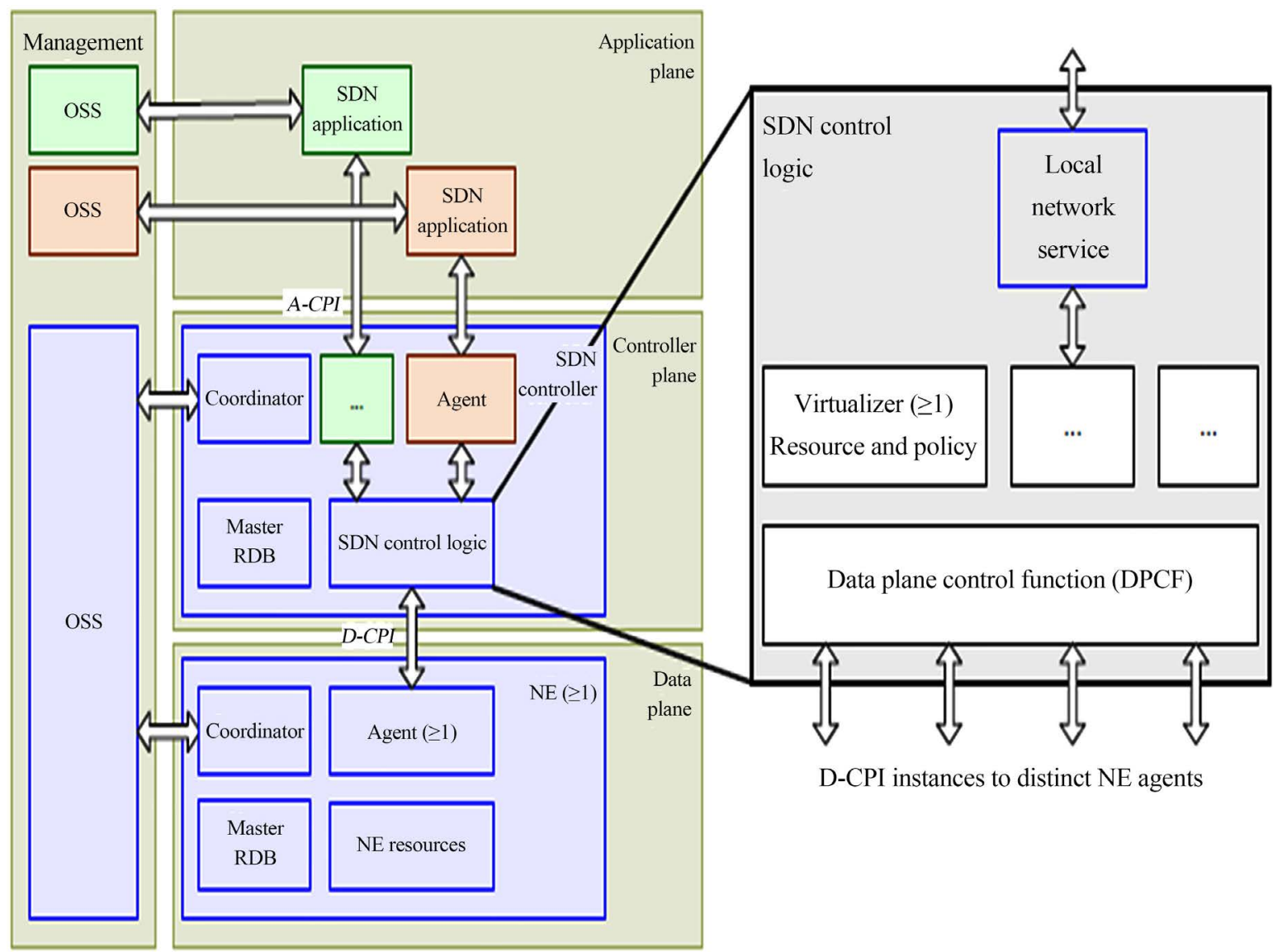

Figure 1. Overview of SDN Architectural plane with the SDN Controller [2] [3].

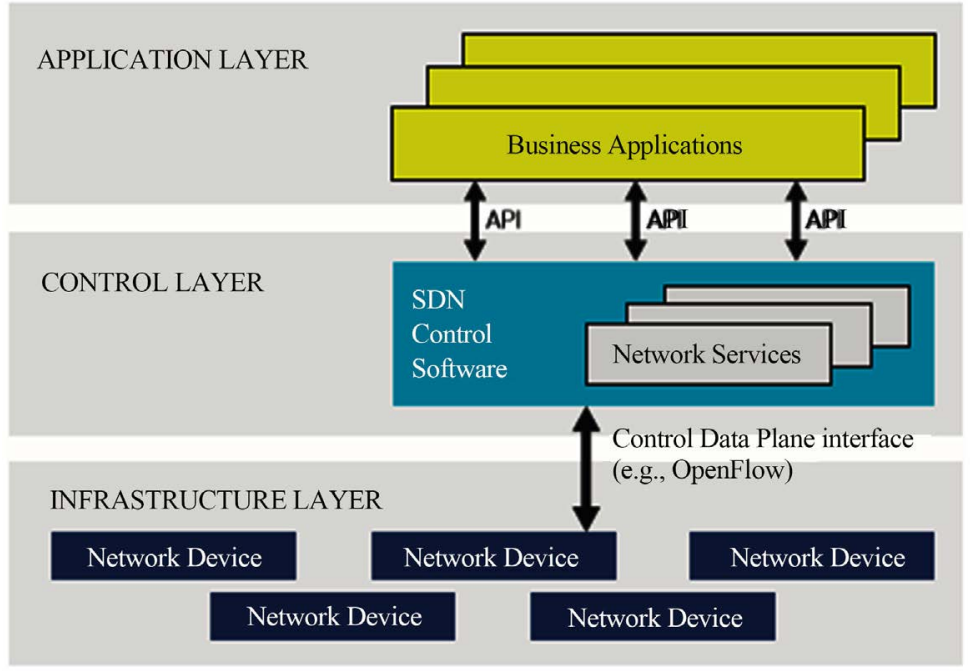

Figure 2. The SDN architecture [4].

\subsection{SDN OpenFlow}

An OpenFlow is a set form of communication standard interface that has the control and ability to forward the layers of SDN architecture. OpenFlow authorizes access to and performs the manipulation of taking forward the plane of 
network devices like routers and switches. The devices can be either physical or virtual (hyopervisor-based). OpenFlow offers security concerns that are man-in-the middle, failure and potential sole point attacks, and issues in programming and communication channels issues [5].

\subsection{Latency}

Before Latency is the period taken by a sent message traversing the system to reach the desired destination. However, handovers in mobile technology are expected to increase as due to the growing trend of better applications in the market such as Skype, Facebook, or MSN Messenger that are also available on mobile phones. The sending and receiving of data can take place even when the applications are inactive. During the sending of data, signals can be lost along the way due to the fluctuations of the waves and other factors that affect data traffic on the network channels.

Generally, there tend to be a collection of components which can result in maximum and higher latency for 3G/4G connections. In case individuals are linking with an occupied or very busy sector in that case the over-the-air network needs to manage through assertion which is actually manages through "scheduling" when every single handset/client can easily dispatch and receive information. Additionally, in case if your own signal is actually poor to or weak through the tower system therefore you need to manage with error correction and re-transmissions.

The second part which could possibly trigger latency is assertion inside the tower system backhaul network and therefore lastly the routing and proxies tips that mobile phone service providers apply in order to facilitate consistent roaming through one tower to other tower (this particular is actually in their unique core) can easily present a considerable amount of latency even when every single thing looks appropriate and excellent. 4G/LTE is actually a "today" advanced cellular networking technology.

Lots of customers of $3 \mathrm{G}$ observe the fact that latency overall performance looks very weak and bad.

With regard to certain programs, a little wait is extremely crucial. Therefore, how actually does Latency in 4G/LTE analyses among elder wireless specifications? Looking at a variety of market reports upon the actual condition of international LTE, particularly the overall performance of mobile phone networks operating a couple fundamental metrics, average download speed and "time on" LTE.

One issues people checked out was exactly how LTE downloading speeds carries out in contrast to some other sorts of wireless technologies, that leaves LTE efficiency in a framework with $3 \mathrm{G}$, and $4 \mathrm{G}$ technologies around the world, in addition to normal Wi-Fi speeds internationally (see Figure 3).

Of course, downloading speed rate really does not even identify the entire facts for mobile phone network performance, however, mainly because it is actually the headline declare that networking sites promote, this is actually the one 


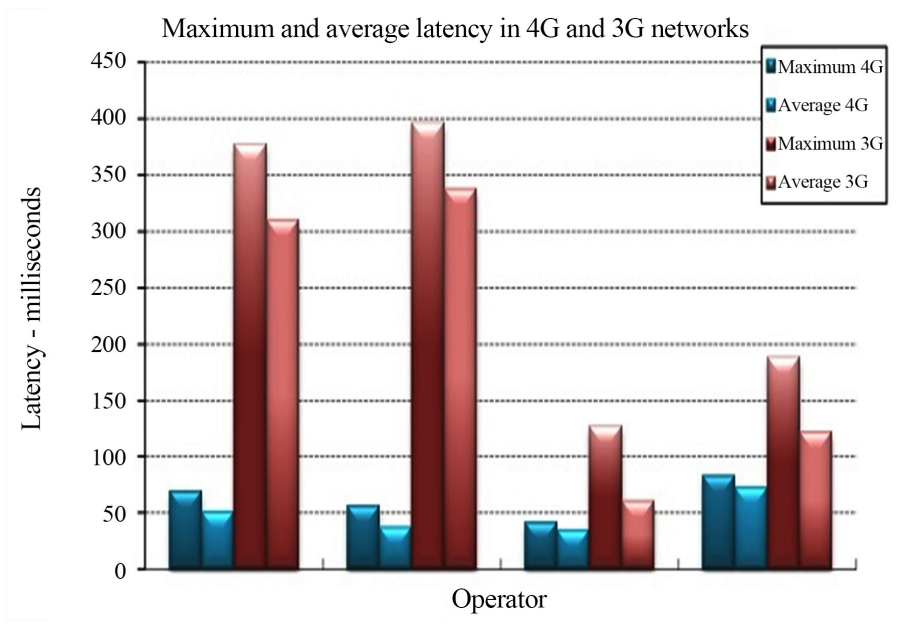

Figure 3. Maximum and average latency in $4 \mathrm{G}$ and $3 \mathrm{G}$ networks [6].

we determined to utilize as ideally consultant of network overall performance. For the mobile phone consumers, upload speed, download speed, and also latency are extremely significant, along with their general significance decided by the particular use a customer applies the system to.

\section{The Handover}

A handover in a networking infrastructure is the transfer or transmission of data or ongoing call on session between two connected channels working under a cellular network. The major reasons why a handover is conducted include:

$>$ The handover can be performed when one of the users is out of range.

$>$ A handover can also be conducted in cases when a base station has accommodated the required users and is full.

A handover is essential as it enables a set latency to occur on server interruption time under a cellular network management on mobile devices.

\subsection{The Requirements of a Handover Framework}

1) Flexibility and latency- The framework must be flexible in manner to enable the accommodation of heavy traffic on the network regardless of the latency performance

2) The networks should be handled using heterogeneous BS entities that involve the pico and femtocells.

3) The ability to accept a handover optimization in a multicarrier device with the capability to optimize the usage of sending cellular data.

\subsection{The Features of a $3 G$ Handover Framework}

1) A handover in a $3 G$ network offers only one ability of Break Before Entry (BBE) operation.

2) The handover selection and initiation under $3 G$ network are rigidly under the control of the network.

3) The handover in a $3 G$ network offers support to a lossless and seamless 
handover.

4) The procedures used by users are defined by are assigned according to the resource bearer.

5) Data is differently handled by the packet data convergence protocol (PDCP) through forwarding data to the eNodeB sides and UE in unique modes than those defined (see Figure 4).

\section{Software Defined Network (SDN) and OpenFlow for 5G}

The 5G (fifth generation) is underway and is expected to be rolling in the market by the year 2020 . The new technology is under scrutiny to ensure that the generation will be efficient and manage to solve issues experienced by the $3 \mathrm{~g} / 4 \mathrm{G}$ networks that are currently in the market. The major target of the $5 \mathrm{G}$ is to enhance a user-centric concept unlike the operator-centric concept used under the $3 \mathrm{G}$ network or the service-centric concept under the 4G network [8] (see Figure 5).

Despite multiple technologies coming in to solve the latencies experienced in communication under $3 \mathrm{G} / 4 \mathrm{G}$ networks, software Defined network (SDN) is the proposed technique in solving the issues experienced.

\subsection{The 5G Mobile Wireless Communication}

Since the research on the $5 \mathrm{G}$ network of communication is under investigations that can be able to use more patterns and other new applications that can be introduced into the market by the time the network will be introduced. The major aims of the network will be an interconnection of more machines, different modules, and actuators that can serve billions of connected clients on the internet using big data applications.

NTT DOCOMO Technical Journal Vol. 11 No. 3

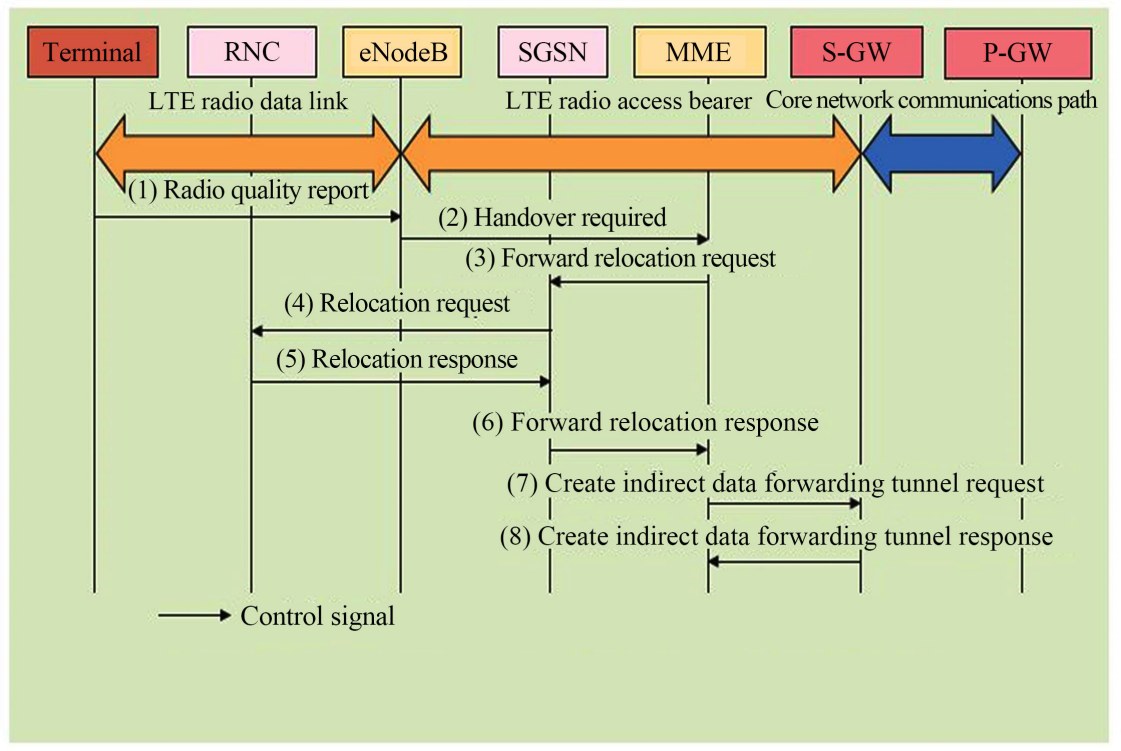

Via: 3g4g.blogspot.com

Figure 4. Preparation Procedure for Handover from LTE to 3G [7]. 


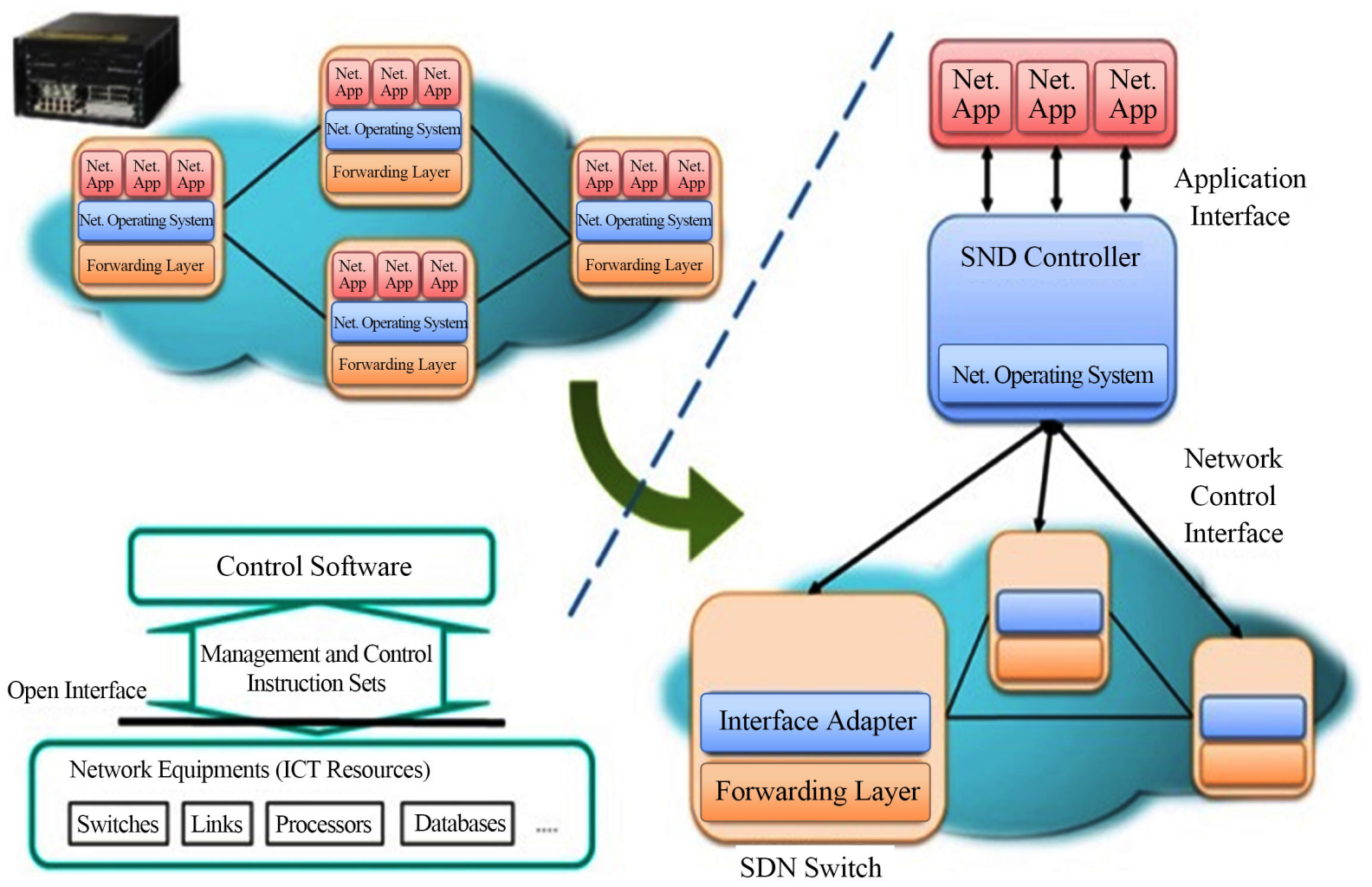

Figure 5. SDN controller and Open Flow model [8].

The big data applications will work better under a modernized and high-speed transmission of traffic and data through the most expected fastest network under the cellular mobile networks. The orientation of the 5G network architecture will be designed to work in a manner as indicated in the diagram below. The importance of SDN inside 5G wireless networks is present particularly inside its capability to supply new functionality such as network virtualization, creating and automating latest facilities on peak of the virtualized strategies, in trusted as well as secure networks. Furthermore, SDN makes it possible for the breakup of the control logic through vendor-specific hardware for start and also vendor-neutral software users.

Therefore, it allows developing data and routing operating operations of wireless structure into program solutions in overall purpose computer or also within the cloud [9] (see Figure 6).

\subsection{Software Defined Network (SDN) for 5G Implementation Background}

The SDN is anticipated to make adjustments to the bandwidth for every radio access points (RAP) to enable the use of a baseband unit (BBU). Apart from the increases usability of the bandwidth, the implementation of the network will be supported with devices that support the transmission of data under the $5 \mathrm{G}$ network to remote regions have over time experienced problems in receiving signals. 


\section{NETWORK ARCHITECTURE FOR 5G MOBILE}
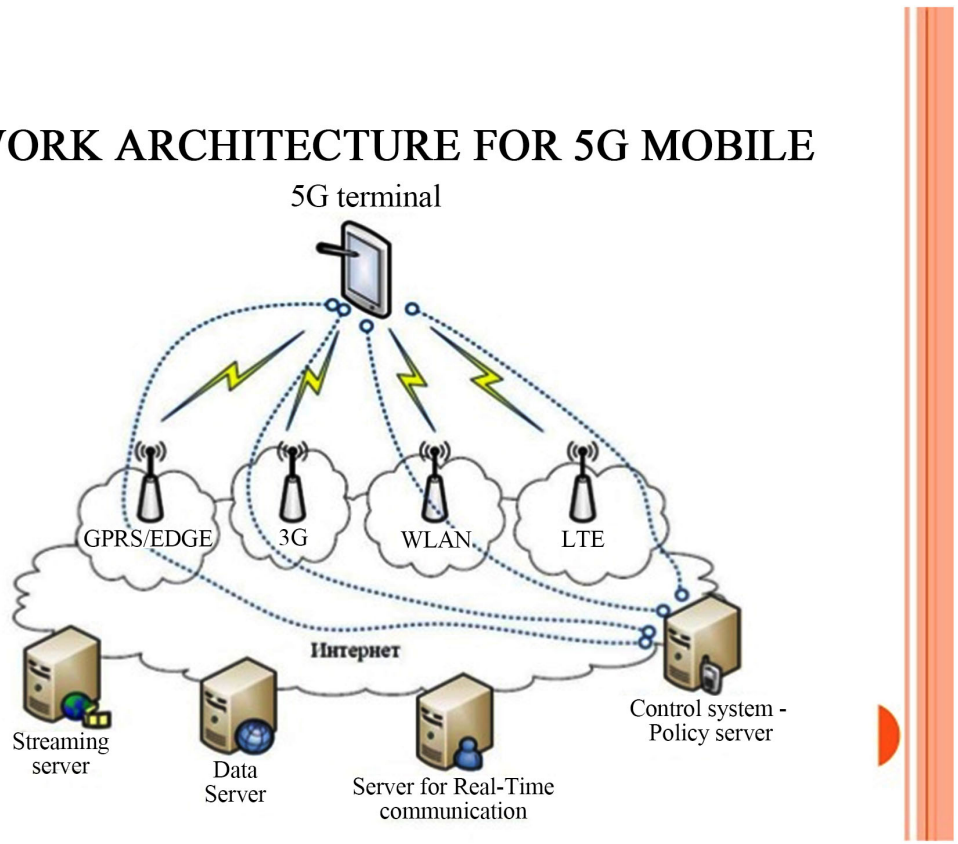

Figure 6. Network architecture for 5G mobile [9].

The devices do not need to be erected in every region but very strong equipment in data transmission over long distances should be incorporated to assure that everyone has been reached out for better communication. The expected connectivity is deemed to be steady among the connected devices of every user and RAP.

\subsubsection{Deploying OpenFlow with Mobile IP}

As identified in [10] Mobile IP network has challenges related to its security issues where when the mobile registers a new care-of-address of a foreign agent to a home agent a malicious attack can occur after registration with the mobile node.

With triangular routing and IP-IP tunneling of the foreign agent and home agent there is a delay and probability of having network congestion. There are handoff issues of mobile devices with the home agent as huge traffic conditions during handoff cannot be handled in this scenario.

The OpenFlow based Mobile IP with routing of packets and handoff are optimized with the controller's application as can also be reviewed with the algorithm mentioned in [10].

The registration process with Open Flow in a Mobile IP network can be further understood in Figure 7 here.

\subsubsection{The Simulation}

Using Mininet 2.2.1 Edit following Model has been developed for the Handover between the Source and Target SDN for Multi SDN Controller domain as the UE or host moves from one SDN to the other with the following Topology diagram at as follows (Figure 8). 


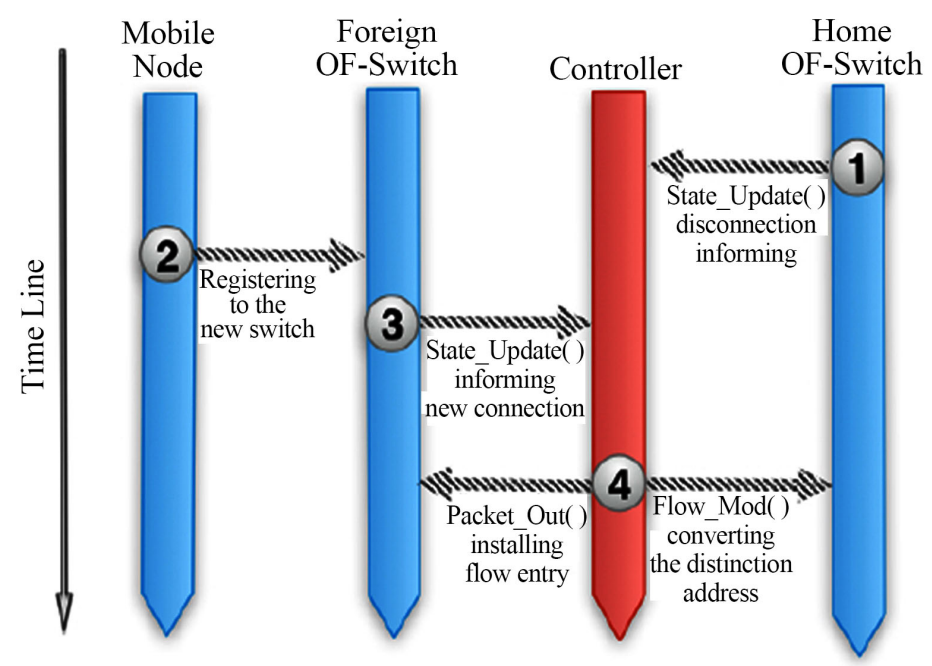

Figure 7. Registration process with OpenFlow in Mobile IP Network [10].

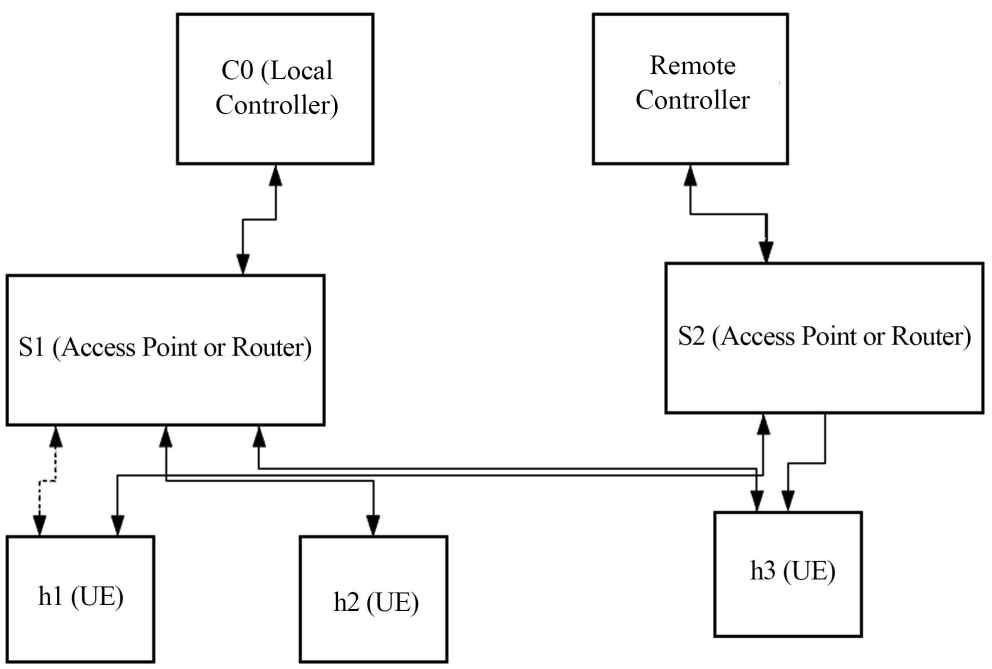

Figure 8. A Tree Topology for Hand Off as host or UE moves from Source SDN to Target SDN Domain with the Southbound API.

The objective of the proposed topology will handle two aspects that firstly the host h1 (UE) is linked to the switch S1 (Access Point or Router) under the source SDN controller C0 (local controller) and S2 (Access Point or Router) Switch under the remote controller. Secondly, on de-linking the host h1 from the switch S1 the host h1 continues to be linked with switch S2 with no change in the IP address. Here the remote controller can control switches and routers in a geographically separated server.

So, at the Southbound API a seamless handoff mechanism can be simulated for the host h1 moving from one SDN Controller to the other. In Southbound API the SDN controller interfaces with the physical hardware of the network like the Access Points, Macro base stations, Switches or Routers.

The algorithm that is deployed for dynamic linking of hosts moving between Multi SDN controllers using South Bound API is as follows in the Mininet: 
1: Create a Network of Multiple Controllers C0, C1 or Remote Controller at different ports

2: Create a Custom Switch () subclass then connect to different controllers

Or

Add Switches S1, S2

3: Add Hosts h1, h2, h3

4: Add links:

S1->h1, h2, h3 and set IP address

5: Create $\mathrm{S} 2$ as mobility switch and attach interfaces

6: Move h1 from C0 to C1

The scenario here includes mobility of UEs or mobile users "sta1" and "sta2" where "sta1" and "sta2" are both linked with "ap1" here:

In Figure 9 once the link is established with the Access point "ap1" with SDN Controller C0 the script "HandOffSDNController.py" further shows the handoff mechanism of the "sta1" moving from "ap1" to "ap2" under the SDN Controller C0. The same can be seen in Figure 10 here.

Here, the position of "stal" changes its position from $(19,38)$ to $(30,60)$ as it moves between the SDN controller C0 to $\mathrm{C} 1$. The source and target SDN controllers in this case are created at local host 127.0.0.1 and there is no change in the IP address of the "sta1" linked with "ap1" as it moves from the SDN controller $\mathrm{C} 0$ to $\mathrm{C} 1$ as can be seen in the Figure 11 here.

\section{Results}

The percentage of the results collected from the network survey indicated that the confidence interval for the network to be effective was $95 \%$.

The data transmission rate would increase by an average of $5 \mathrm{Mbps}$ that will enable faster and convenient communications. Since the speed will be too high resulting to more usage of cellular data, during inactive period the network will not be able to transmit data but under the settings options by the user.

The mode of design the $5 \mathrm{G}$ network will operate will enable users to preserve their data for the active sessions with their gadgets.

The simulation for Multi SDN Handoff Mechanism in Mininet 2.2.1 indicates that it is possible to achieve user mobility with the host $\mathrm{h} 1$ or UE moving from one switch S1 to another switch S2 of another domain with no impact or changes in the IP address. Also, Miniedit can be used to build topology and generate scripts to verify mobility on the CLI interface.

In this case since there is no triangular routing method, latency issues are improved as compared to the Mobile IP.

\section{Discussion}

As much as $3 \mathrm{G}$ and $4 \mathrm{G}$ have enabled people to get increased browsing speeds that the $2 \mathrm{G}$ network that was earlier in the market, the improved technology has enabled researchers to come up with methods that can help to generate more traffic and manage big data flows within the shortest time possible without buffering. 


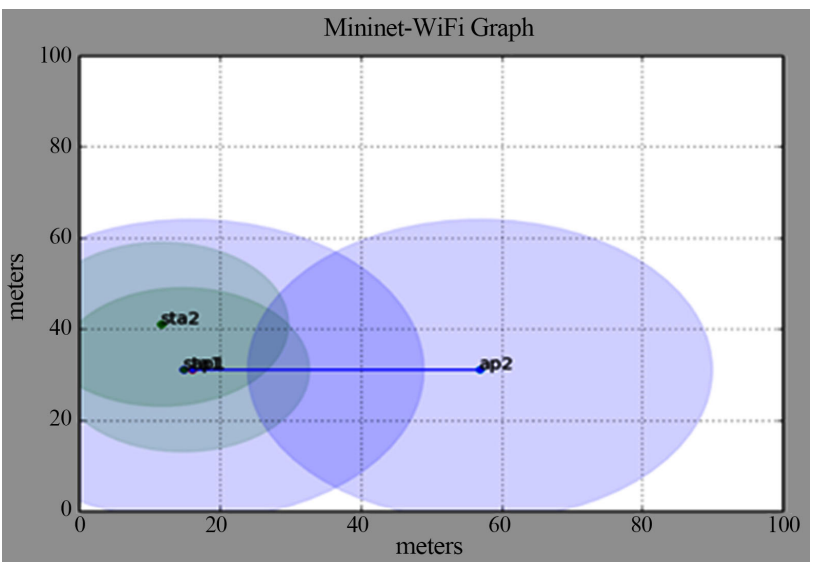

Figure 9. Topology generated with "sta1" and "sta2" linked with ap1 with C0.

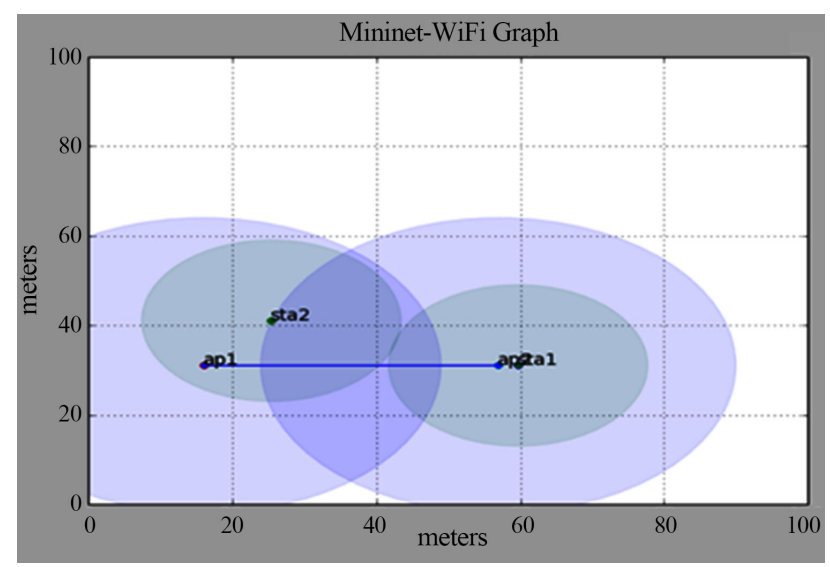

Figure 10. "sta1" moving from the "ap1" to "ap2" with the controller C1.

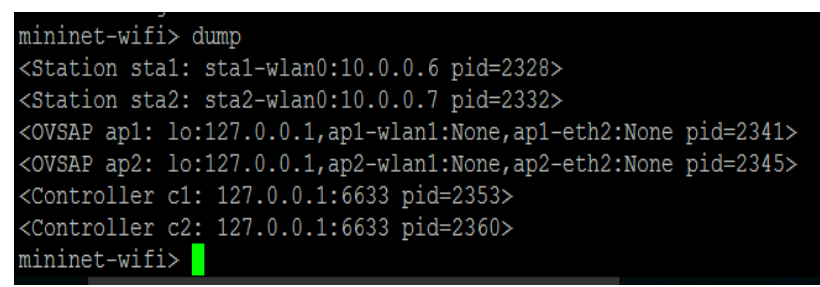

Figure 11. IP address of the Mobile Station "sta1" at 10.0.0.6.

As explained and seen in the results of the $5 \mathrm{G}$ launch that is expected to come into the market by 2020, the world will be expecting the internet service providers (ISP) to lower the data transmission charges because of the increased efficiency of the network. However, before the launch, something must have been agreed upon among the involved parties.

Apart from featuring mobile gadgets such as android phones, the use of tablets and laptops will be under review to include the ability to connect to the internet using cards that can be purchased for specific durations and toped up with credit amounts after the units get exhausted [6]. 


\section{Conclusions}

Many scholars are on the run to provide the world with more reliable and faster 5G wireless network. The software Defined network (SDN) offers virtualization and it has enabled SDN to be the most attracting point by many researchers. More designs of presenting the $5 \mathrm{G}$ network into the market are underway, as the launch has not been done.

The expected high number of devices and applications will open way for new inventors of applications and devices to gain market for their works. The use of local area network (LAN) under a larger number of users will be a must use by every user in the platform of the new wireless generation that can offer the use of more applications and devices connection.

Throughout this report, we have actually discovered various limitations and capacities of SDN-enabled mobile systems and effect on 5G network. We have observed the condition of the artwork in establishing OpenFlow to LTE systems.

A bunch of benefits as well as a few open problems had been reviewed; however, this is actually very clear that SDN is actually the normal development for mobile phone channels.

To tackle the shortage of a flexible and reliable software device which can possibly be applied for efficiency assessment, we suggested a unique and latest OpenFlow 1.3 component for the ns-3 network simulator which we can use for $5 \mathrm{G}$ technology.

This particular component can easily be applied collectively with LTE component to measure unique structural suggestions. As long term and future services, we will likely examine latest techniques to enhance network QoS and customer skills and experiences.

\section{Conflicts of Interest}

The authors declare no conflicts of interest regarding the publication of this paper.

\section{References}

[1] https://tools.ietf.org/html/rfc5944

[2] Li, Q.C., et al. (2014) 5G Network Capacity: Key Elements and Technologies. IEEE Vehicular Technology Magazine, 9, 71-78. https://doi.org/10.1109/MVT.2013.2295070

[3] Nguyen, X.-N., et al. (2016) Rules Placement Problem in Openflow Networks: A Survey. IEEE Communications Surveys \& Tutorials, 18, 1273-1286. https://doi.org/10.1109/COMST.2015.2506984

[4] Sahoo, K.S., et al. (2016) A Comprehensive Tutorial on Software Defined Network: The Driving Force for the Future Internet Technology Proceedings of the International Conference on Advances in Information Communication Technology \& Computing, ACM, August 2016, Article No.: 114, 1-6. https://doi.org/10.1145/2979779.2983928

[5] Yan, Q., et al. (2016) Software-Defined Networking (SDN) and Distributed Denial 
of Service (DDoS) Attacks in Cloud Computing Environments: A Survey, Some Research Issues, and Challenges. IEEE Communications Surveys \& Tutorials, 18, 602-622. https://doi.org/10.1109/COMST.2015.2487361

[6] Top Optimized Technologies "Performance Analysis of LTE Deployments in Madrid”, Sep. 2013, White Paper, C/Gobelas 17, BajoMadrid, Spain.

[7] Ghadialy, Z. (2011) LTE to 3G Handover Procedure and Signalling. Thursday, 3 March.

[8] Yazıc1, V., Kozat, U.C. and Sunay, M.O. (2014) A New Control Plane for 5G Network Architecture with a Case Study on Unified Handoff, Mobility, and Routing Management. IEEE communications magazine, 52, 76-85.

https://doi.org/10.1109/MCOM.2014.6957146

[9] Sama, M., et al. (2015) Software-Defined Control of the Virtualized Mobile Packet Core. IEEE Communications Magazine, 53, No. 2. https://doi.org/10.1109/MCOM.2015.7045398

[10] Pupatwibul, P., Banjar, A., Al Sabbagh, A. and Braun, R. (2013) Developing an Application Based on OpenFlow to Enhance Mobile IP Networks. Conference on Local Computer Networks, LCN, Sydney, 21-24 October 2013, 936-940.

https://doi.org/10.1109/LCNW.2013.6758535 